\title{
Role of Intravenous Methylprednisolone in Moderate to Severe Thyroid Associated Orbitopathy (TAO) in Indians: A Tertiary Care Institute Study
}

\author{
Shweta Gupta, Manpreet Singh*, Manpreet Kaur, Deepti Yadav, Himanshi Singh, Zoramthara Zadeng and Pankaj \\ Gupta
}

Department of Ophthalmology, Post Graduate Institute of Medical Education and Research, India

*Corresponding author: Manpreet Singh, Assistant Professor, Department of Ophthalmology, Advanced Eye Centre, Post Graduate Institute of Medical Education and Research, Chandigarh, India.

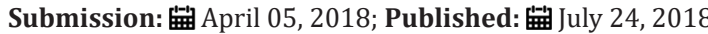

\begin{abstract}
Purpose: Aim was to report outcome in 18 patients of moderate to severe thyroid associated orbitopathy (TA0) treated with intravenous steroids (methylprednisolone).

Methods: Eleven males and 7 females with mean age of 52.92 years received methylprednisolone for TAO. Examination revealed decreased vision(15), lid inflammation(15), \& movement restriction(16). They were hyperthyroid(8), hypothyroid(3), euthyroid(7), \& 8 had pre-existing thyroid disease. Field defects(8), apical crowding(3) and corneal involvement(4) were present. Treatment regime was, regular for 3 days(10), pulse therapy(5) \& combined(3). Response to treatment was evaluated by VISA classification.
\end{abstract}

Results: Improvement was seen in vision(14), inflammation(17), movement(16) and appearance(15). Determinants of poor outcome were, diabetic status, corneal involvement, raised intraocular pressure, preexisting thyroid disease and pulse therapy.

Conclusion: Intravenous methylprednisolone is safe \& efficacious in improving TAO in majority of acute cases along with control of co-morbid conditions.

Keywords: Thyroid associated orbitopathy; Proptosis; Intravenous steroids; Methylprednisolone

\section{Introduction}

Thyroid Associated Orbitopathy (TAO) is the most common cause of bilateral and unilateral (asymmetric) proptosis in adults [1-3]. In almost $90 \%$ patients, it is associated with hyperthyroidism while euthyroid (6\%), Hashimoto's thyroiditis (3\%) and primary hypothyroidism (1\%) constitute the rest.[1-5]. Approximately half of Graves' disease (ophthalmopathy, hyperthyroidism and pretibial myxedema) patients have ocular involvement, which is usually mild. Though, moderate to severe TAO occurs in $20-30 \%$ patients, it is the sight-threatening disease (3-5\%) which require emergency intervention $[2,3,5,6]$. Steroids form the first-line of treatment for severe and active TAO. Many studies have documented the effectiveness of steroids via oral, intravenous or peribulbar route, but the optimal delivery method with minimal side effects remains indeterminate [4-10]. Although, the intravenous route is considered as more effective and better tolerated than the others, few lethal adverse events have been reported with it. $[6,7,8]$ The aim of present study is to analyze the efficacy and safety of intravenous methylprednisolone (IVMP) administered via three different protocols in the patients with sight threatening TAO.

\section{Methods}

We included 18 patients of previously untreated and active TAO from the oculoplastics clinic between June 2014 and April 2016. All patients met the inclusion criteria of, moderate to severe TAO (moderate/severe soft tissue involvement, eyelid retraction $>2 \mathrm{~mm}$, exophthalmos $>3 \mathrm{~mm}$, diplopia) and sight threatening TAO (optic neuropathy, exposure keratopathy). Thorough ophthalmological examination (including intraocular pressure and Schrimer's test) was done at the first visit. Proptosis was measured with the Hertel's exophthalmometer.

Soft tissue involvement was documented with reference from the color atlas by EUGOGO (European group on Graves' orbitopathy) [8]. the assessment of soft-tissue disease activity was carried out using the seven points of clinical activity score (CAS)- spontaneous retrobulbar pain, pain during eye movements, conjunctival redness, chemosis, redness of eyelids, swelling of eyelids, swelling of caruncle. Each one is given 1 point and the final score was calculated [9]. Eye muscle involvement was assessed using the Gorman 
diplopia score- absent (no diplopia), intermittent (when patient is tired), inconstant (at extremes of gaze) and constant (continuous diplopia in primary and reading position) [10]. VISA classification was used for the analysis of improvement in Vision, Inflammation, Strabismus and Appearance [11]. Visual fields (VF) assessment was done using Humphery's VF analyser following 76-2 SITA standard strategy.

Complete blood biochemical profile (blood sugar levels, liver function tests etc.) and thyroid function tests were requested. Endocrinology consultation and blood pressure monitoring was done before starting the treatment. Informed consent was obtained from all patients. Patients received IVMP in three different protocols- a) 1 gm IVMP for three consecutive days ( $\mathrm{n}=10$ patients), 500mg IVMP/ week for 6 weeks followed by 250mg IVMP/week for 6 weeks ( $n=5)$ and combined treatment $(\mathrm{n}=3)$. After the completion of intravenous regimens, all patients were started on oral prednisone- $40 \mathrm{mg} /$ day for 1 st week, $30 \mathrm{mg} /$ day for the $2 \mathrm{nd}, 20 \mathrm{mg} /$ day for the $3 \mathrm{rd}$ and $10 \mathrm{mg} /$ day for the $4 \mathrm{th}$ week. This sequence of therapy was repeated each month for the next 2 months i.e. in each of the following protocols IVMP was then followed by tapering doses of oral prednisone for 6-8weeks. The cumulative dose was $9 \mathrm{~g}$ (not exceeding $10 \mathrm{~g}$ ).

Ophthalmological assessment with VISA scoring was performed at 1 week, 1 month, 3 month and 6 months of followup visits. Side effects of steroid therapy and biochemistry analysis were evaluated and recorded at each followup. After 6 months of followup, a satisfactory response to the therapy was defined as a relevant improvement in at least two major and one minor criterion. Major criteria being a decrease of $\geq 2 \mathrm{~mm}$ each in proptosis and lid width

Table 1: Demographics and clinical data of patients.

\begin{tabular}{|c|c|}
\hline Mean age & $52.92 \pm 11.7$ years (range, $32-75$ years) \\
\hline Female: male & $7: 11(\mathrm{~F}-38.8 \%)$ \\
\hline Unilateral : Bilateral & 09:09 \\
\hline Duration of thyroid disease (months) & $56.5 \mathrm{~m}$ [1 month-30 years] \\
\hline Duration of TAO (months) & $3.6 \mathrm{~m}$ [2 days- 2 years] \\
\hline \multicolumn{2}{|c|}{ Thyroid disease } \\
\hline Graves' disease & $8(44.44 \%)$ \\
\hline Hashimoto thyroiditis & $3(16.66 \%)$ \\
\hline Euthyroidism & $7(38.8 \%)$ \\
\hline \multicolumn{2}{|c|}{ Associated systemic disease } \\
\hline Diabetic & $4(22.22 \%)$ \\
\hline HTN & $7(38.8 \%)$ \\
\hline $\mathrm{F} / \mathrm{h} / \mathrm{o}$ of thyroid disease & $1(5.55 \%)$ \\
\hline Smoking & $1(5.55 \%)$ \\
\hline Associated dermopathy/Autoimmune disease & None \\
\hline
\end{tabular}

At the end of 6 months, visual acuity improved in 11 (61.1\%), remained stable in $6(33.3 \%)$ and deteriorated in 1 (5.55\%). Visual fields defects $8(44.4 \%)$, relative afferent pupillary defect 5 $(27.7 \%)$ and disc edema $3(16.6 \%)$ resolved completely. Diplopia in degree of diplopia, reduction of $\geq 2$ points of CAS and changes of $\geq 1 / 10$ in visual acuity and minor criterion were soft tissue changes, self assessment evaluation [12].

The normality assumption for continuous variables was evaluated by the Kolmogorov-Smirnov test. Continuous variables were presented as means and standard deviations for normally distributed variables. They were compared using the one-way Student's t test for paired samples, or its non-parametric equivalent, the Wilcoxon matched-pair signed rank test. Categorical variables are presented as counts and percentages and were compared with the chisquare test or McNemar's test for repeated measurements. To test parameters independently associated with treatment response, variables (age, gender, smoking, duration of TAO) multivariable regression was used. For all analyses, a two-sided $\mathrm{p}<0.05$ was considered statistically significant.

\section{Results}

The demographic data is briefed in Table 1. Most common presenting symptom was globe protrusion $(n=9)$ followed by eyelid swelling ( $n=7)$, redness \& watering ( $n=5$ each), diplopia $(n=4)$ and diminution of vision \& ocular pain ( $\mathrm{n}=2$ each). The foreign body sensation and drooping of eyelid was complained by one each. On examination, conjunctival inflammation $(n=18)$, eyelid signs $(n=15)$, eyelid inflammation $(n=15)$ caruncle inflammation $(n=5)$ and blepharoptosis $(n=1)$, were noted. Hormonal therapy was monitored by the endocrinologist in all and control of thyroid hormone levels was achieved during the treatment course. The intravenous steroids were administered to all followed by oral steroid therapy according to the mentioned protocols.

restricted EOM and raised RBR resolved in all. Differential IOP improved in $6(54.54 \%)$, deteriorated in $2(18.18 \%)$ while no change was observed in $3(27.27 \%)$ patients. Four (22.2\%) patients had exposure keratopathy which resolved after treatment in two. Re- 
duced Schirmer's test values $(<5 \mathrm{~mm} / 5$ minutes) were noticed in 7 (38.8\%) which improved after steroid therapy in 5 patients.

The proptosis was also significantly reduced at the end of treatment (Table 2). However, abnormal exophthalmometer readings ( $>19 \mathrm{~mm}$ ) persisted at the end of followup in $13(72.22 \%)$ patients. The mean CAS value reduced significantly in 17 (94.4\%) patients while in one (5.5\%), it deteriorated. Hence, Vision improved in 11
$(61.1 \%)$ patients, remained stable in $6(33.33 \%)$ and deteriorated in 1 (5.55 \%). Inflammation was significantly reduced in 17 (94.44\%) patients while it persisted in one (5.55\%). Clinical improvement in strabismus was noticed in $100 \%$. Fourteen (77.77\%) patients improved in appearance while deteriorated in 1 (5.55\%). According to the defined criteria, 17 out of 18 patients (94.4\%) demonstrated treatment response.

Table 2: Change in CAS, proptosis and ocular pressure before and after therapy.

\begin{tabular}{|c|c|c|c|}
\hline & Before steroids & After steroids & p value \\
\hline Proptosis $(\mathrm{mm})$ & $4.70 \pm 1.83$ & $2.87 \pm 2.20$ & $\mathrm{P}<0.05$ \\
\hline IOP $(\mathrm{mmHg})$ & $20.8 \pm 8.12$ & $18.9 \pm 6.76$ & Not significant \\
\hline CAS & $4.05 \pm 1.22$ & $1.38 \pm 1.06$ & $\mathrm{P}<0.05$ \\
\hline
\end{tabular}

During the 6 months followup, only 1 (5.55\%) patient had a relapse which was treated with other immunotherapeutic drugs. Overall, 4 $(22.22 \%)$ patients developed steroids related side-effects with 3 having raised IOP. One patient developed acute psychosis for which the patient was treated at psychiatry department and resolved completely in 5 days. Clinically insignificant rise in blood pressure was observed in 3 patients. There was no significant weight gain (increase of $\geq 3 \mathrm{~kg}$ ) or raised blood glucose, serum lipids and aminotransferases levels in the study population over 6 months of followup. No other systemic or ocular complications were observed during this period.

\section{Discussion}

This was a retrospective analysis of efficacy and tolerability of a combined parenteral and oral steroid therapy in 18 patients with untreated and active TAO. The present study also evaluated the affect of parameters such as age, gender, smoking, duration of hyper- thyroidism, duration of TAO and CAS on the response to steroids. We have demonstrated that combined parenteral and oral steroid therapy is effective and safe in the treatment of TAO.

The response rate in our study group was $94.4 \%$. Intravenous steroids showed the greatest effectiveness on soft tissue changes (clinically evaluated by CAS), whereas the reduction in proptosis was not significant. Randomized and nonrandomized trials have reported an average reduction in CAS by 2.5 and 3.5 points, respectively $[9,12-15]$. The inactivation of Graves' orbitopathy was shown to be in $59 \%$ and $89 \%$ by these trails, respectively [16-21]. Our results showed a significant decrease of 2.6 points in CAS which is in concordance with previous reports. Belesin et al. [22] showed better treatment response $(80 \%)$ in patients with CAS $>4$ as compared to those having CAS $<4$ (23\%), p<0.05 [22] (Table 3). In our study, all patients had CAS of $>4$ which according to multivariable regression analysis, is the only independent parameter associated with positive treatment response $(\mathrm{p}<0.001)$ [9-15].

Table 3: Comparison of our study with that of Belesin et al. [22]

\begin{tabular}{|c|c|c|}
\hline & Our study & Belesin, et al. [22] \\
\hline No. of patients(n) & 18 & 40 \\
\hline Age(yrs) & $52.92 \pm 11.7$ & 6 \\
\hline Evaluation period (m) & 3 & 500 mg for 2 alternate days* $6 \mathrm{~m}$ \\
\hline IV methylpred regime & 19*3 days 250 mg wkly *6 wks Combined \\
\hline Improvement in VA & & $36 \%$ \\
\hline Decrease in mean CAS & $61.10 \%$ & 4.5 to 2 \\
\hline Improvement & 4.0 to 1.38 & $37(74 \%)$ \\
\hline No change & $16(94.4 \%)$ & $13(26 \%)$ \\
\hline Deterioration & Nil & Nil \\
\hline Diplopia improvement & $1(5.55 \%)$ & $21(42 \%)$ \\
\hline Overall Response & $4(100 \%)$ & $33(66 \%)$ \\
\hline Side effects & $17(94.4 \%)$ & $35(70 \%)$ \\
\hline Relapse & $2(11.1 \%)$ & $2 / 33(6 \%)$ \\
\hline
\end{tabular}


After completion of treatment, the proptosis did not change significantly in our study but the improvement of at least $2 \mathrm{~mm}$ occurred in $9(50 \%)$ patients. This is similar as compared with various previous studies $[13,14]$ while few showed a better response [15,16,17-21]. Steroids have anti-inflammatory and immunosuppressive actions which has favorable effect during the active phase of TAO. High CAS, high TSHRAb levels and shorter duration of orbitopathy are well known parameters of TAO activity and predictors of therapeutic efficacy [18-22]. In our patients the response to IVMP therapy was influenced by long duration of thyroid disease, associated diabetes mellitus, high intraocular pressure, smoking behavior and pulse therapy, which were found to be associated with the patients, who responded suboptimally to the treatment.

Smoking affects the pathogenetic immunological reaction as it alters the structure of thyrotropin receptor, making it more immunogenic, leading to increased production of thyrotropin receptor stimulating antibodies. These antibodies act prominently with the antigens of retro-orbital tissue [17-22,23]. In addition; smoking reduces the effectiveness of glucocorticoids in a dose-dependent manner during treatment [18,23]. Unlike Kahaly et al. [19] who found that the females and the patients younger than 50 years of age, responded better to steroid therapy, we did not find any impact of gender and age on the response to steroid therapy [16]. Adverse events are a major concern of steroid therapy with reported morbidity and mortality rates of 6.5 and $0.6 \%$, respectively [6].

There are numerous case series and reports on the potential serious hepatic, cardiovascular and cerebro-vascular effects with fatal outcome [6]. In our study, blood sugar levels, liver enzymes and blood pressure was monitored on every visit and was within the normal range in all patients. One patient who developed psychosis has been documented as serious side effects [7]. All other side effects were non-severe and resolved with treatment. Belesin et al. [22] found that weight gain and hirsutism are the most common side effects, whereas serious side effects depend on preexisting disease as well [22]. In our study all patients had significant improvement in the Quality of life related to vision, physical (symptoms) and psychological function after the treatment.

For steroid administration, the intravenous route is reported to be more effective and better tolerated than the oral route $[15,16,23]$. However, deterioration of vision is a common issue after discontinuation of pulse steroid therapy. A recent multicenter study described relapse in 33\%, 21\% and $40 \%$ corresponding to different cumulative doses of $2.25 \mathrm{~g}, 4.98 \mathrm{~g}$ or $7.47 \mathrm{~g}$, respectively over 12 weekly infusions. The relapse rate of our study was $5.55 \%$. The inter-pulse oral prednisone might reduce the recurrence rate and stabilize the overall recovery in patients with TAO. Common protocol use a $4.5 \mathrm{~g}$ cumulative dose of methylprednisolone subdivided into 12 weekly infusions $[16,23]$.

Three days high dose IVMP 3 protocol is practically more convenient than the weekly IVMP protocol especially for the patients coming from far peripheral areas to get treatment from a tertiary care referral institute. Moreover, it seems to be safer option con- sidering the possibility of side effects while conducting clinical and biochemical evaluation. Though the number of patients is small, the follow-up is short and the study design is retrospective, ours is the first Indian study evaluating the effects and safety of different intravenous steroid protocols in patients having severe/ sight threatening TAO. A well controlled, double blinded, randomized, prospective study is needed in Indian patients which will give further insight in this complete scenario.

\section{Conclusion}

In conclusion, Intravenous methylprednisolone is a safe and efficacious drug to be used in severe/ sight threatening acute TAO patients. In the patients' suffering from co-morbidities, an adequate control is enough for its safe administration. Thorough clinical evaluation, close supervision while administering the drug and proper follow-up monitoring of biochemistry, is essential. Apt patient selection based on disease severity and the potential life-threatening side effects from high dose steroid therapy is warranted.

\section{References}

1. Kriss JP, Pleshakov V, Rosenblum AL, Holderness M, Sharp G, et al. (1967) Studies on the pathogenesis of the ophthalmopathy of Graves' disease. J Clin Endocrinol Metab 27(4): 582-593.

2. Burch HB, Wartofsky L (1993) Graves ophthalmopathy: current concepts regarding pathogenesis and treatment. Endocr Rev 14(6): 747-793.

3. Bahn RS (2010) Graves' ophthalmopathy. N Engl J Med 362(8): 726-738.

4. Zang S, Ponto KA, Kahaly GJ (2011) Intravenous glucocorticoids for Graves' orbitopathy: Efficacy and morbidity. J Clin Endocrinol Metab 96(2): 320-332.

5. Bartalena L, Krassas GE, Wiersinga W (2012) Efficacy of three different cumulative doses of intravenous methylprednisolone for moderate to severe and active Graves' orbitopathy. J Clin Endocrinol Metab 97(12): 4454-4463.

6. Marinó M, Morabito E, Brunetto MR, Bartalena L, Pinchera A, et al. (2004) Acute and severe liver damage associated with intravenous glucocorticoid pulse therapy in patients with Graves' ophthalmopathy. Thyroid 14(5): 403-406.

7. Sisti E, Coco B, Menconi F, Leo M, Rocchi R, Latrofa F et al. (2015) Intravenous glucocorticoids therapy for Graves' ophthalmopathy and acute liver damage: an epidemiological study. Eur J Endocrinol 172(3): 269-376.

8. Bartalena L, Baldeschi L, Dickinson AJ, Eckstein A, Kendall-Taylor P, et al. (2008) Consensus statement of the European group on Graves orbitopathy (EUGOGO) on management of Graves orbitopathy. Thyroid 18(3): 336-346.

9. Mourits MP, Prummel MF, Wiersinga WM, Koornneef L (1997) Clinical activity score as a guide in the management of patients with Graves ophthalmopathy. Clin Endocrinol (Oxf) 47(1): 9-14.

10. Dickinson AJ, Perros P (2001) Controversies in the clinical evaluation of active thyroid associated orbitopathy: use of a detailed protocol with comparative photographs for objective assessment. Clin Endocrinol (Oxf) 55(3): 283-303.

11. Dolman PJ, Rootman J (2006) VISA Classification for Graves' orbitopathy. Ophthalmic Plastic Reconstructive Surgery 22(5): 319-324.

12. Bartalena L, Pinchera A, Marcocci C (2000) Management of Graves ophthalmopathy: reality and perspectives. Endocr Rev 21(2): 168-199. 
13. Kendall-Taylor P, Crombie AL, Stephenson AM, Hardwick M, Hall K (1998) Intravenous methylprednisolone in the treatment of Graves ophthalmopathy. Br Med J 297(6663): 1574-1578.

14. Bahn RS, Gorman C (1987) Choice of therapy and criteria for assessing treatment outcome in thyroid-associated ophthalmopathy. Endocrinol Metab Clin N Am 16(2): 391-407.

15. Bartalena L, Marcocci C, Bogazzi F, Luca Manetti, Maria Laura Tanda, et al. (1998) Relation between therapy for hyperhyroidism and the course of Graves ophthalmopathy. N Engl J Med 338: 73-78.

16. Kendall-Taylor P, Crombie AL, Stephenson AM, Hardwick M, Hall K (1998) Intravenous methylprednisolone in the treatment of Graves' ophthalmopathy. Br Med J 297(6663): 1574-1578.

17. Kaupinen-Makelin R, Karma A, Leinomen E, Löyttyniemi E, Salonen 0 , et al. (2002) High dose intravenous methylprednisolone pulse therapy versus oral prednisone for thyroid associated ophthalmopathy. Ophthalmol Scand 80(3): 316-321.

18. Marcocci C, Bartalena L, Tanda LM, et al. (2001) Comparison of the effectiveness and tolerability of intravenous or oral glucocorticoids associated with orbital radiotherapy in the management of severe Graves' ophthalmopathy: results of a prospective, single-blind, randomized study. J Clin Endocrinol Metab 86(6): 3562-3567.

19. Kahaly GJ, Pitz S, Hommel G, Dittmar M (2005) Randomized, single blind trial of intravenous versus oral steroid monotherapy in Graves' orbitopathy. J Clin Endocrinol Metab 90(9): 5234-5240.

20. Utiger RD (1998) Effects of smoking on thyroid function. Eur J Enocrinol 138: $368-369$

21. Hegedius L, Brix TH, Vestergaard P (2004) Relationship between cigaretes smoking and Graves ophthalmopathy. J Endocrinol Invest 27(3): 265-271

22. Beleslin BN, Ciric J, Zarkovic M, Stojkovic M (2014) Efficacy and safety of combined parenteral and oral steroid therapy in Graves' orbitopathy. Hormones 13(2): 222-228.

23. Perumal B, Meyer DR (2015) Treatment of severe thyroid eye disease: a survey of the American Society Ophthalmic Plast Reconstr Surg 31(2): 127-131. (c) (i) Creative Commons Attribution 4.0 International License

For possible submissions Click Here
Submit Article

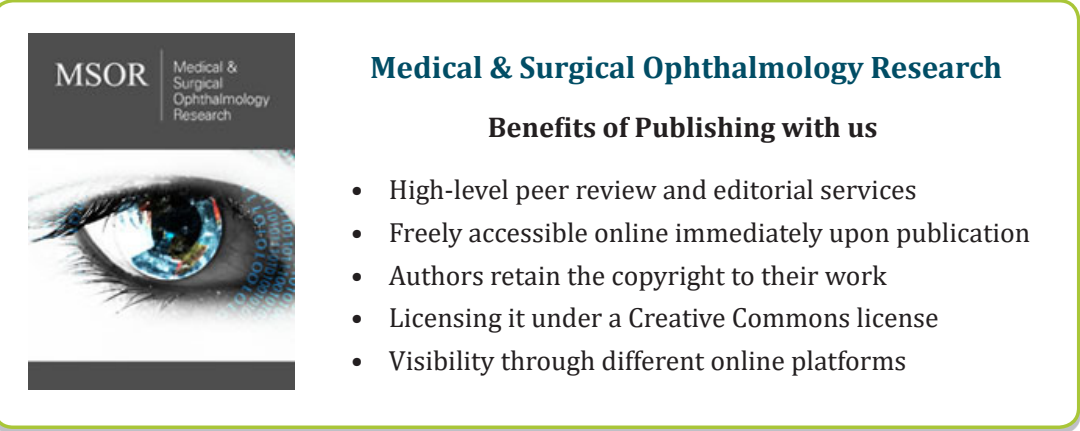

